\title{
Our services and processes are opening up
}

\author{
We encourage openness in publishing, and promote increased transparency in peer review.
}

W hat do you have to hide? The 'nothing to hide' argument most often used when debating privacy - is also fitting when discussing the processes of scientific research and scientific assessment. Accelerated by the growth of the Internet, by the open-access movement, and now by the COVID-19 pandemic, the trend towards openness in the doing and publishing of research (through open lab notebooks, open data, open code, protocol sharing, preprinting, open-access publication and open commenting, in particular) is unquestionable. The fundamental arguments are powerful: faster scientific progress, the promotion of collaboration, increased trust and wider accessibility.

Yet lofty arguments for openness especially for unconditional transparency - don't always lead to fair policies and best outcomes for the common good. Three themes are particularly illustrative in this regard. First, research is not only highly collaborative, it is also highly competitive, and increasingly so. Limited resources must be allocated wisely, largely on the basis of achievements, reputation built on solid work, and promise (of individuals, teams and research fields). Healthy balances of collaboration and competition and of trust and scepticism (Nat. Biomed. Eng. 3, 159-160; 2019) drive science forward. Unconditional (or mandated) openness can, however, tilt such evenness towards insufficient competition or scepticism. For example, forcing criticism to be fully open (by disincentivizing anonymity during peer review and in post-publication peer review) can muzzle necessary dialogue. And publishers and preprint services with inadequate or insufficient levels of vetting strengthen the spread of misinformation; social media, and social-media platforms, have exacerbated this.

Second, fair and optimal allocations of resources for research requires metrics of evaluation. Transparency and openness about the criteria for such assessments (most notably of grant applications and papers, and also in hiring), and how they are carried out, builds trust. But forcing undue openness in the publication of assessment outcomes can undermine it: reputations are not built by making failures public. And because judgments on imperfect information and on complex or undetermined outcomes are rarely purely objective, opening up subjectivity to wider debate (particularly before decisions are taken) can be unproductive.

Third, the rapid and open sharing of research outputs does indeed speed up progress - especially when data, code and protocols are readily shared (Nat. Biomed. Eng. 4, 845-846; 2020) alongside preprint deposition. Yet paid open access for peer-reviewed papers creates imbalances by shifting the cost burden from consumers of research to its fewer producers. It can also create perverse incentives that result in insufficient vetting (prominently by predatory journals). And when open access is mandated, it restricts access to publishing in costly journals to those who can afford it.

However, that there are unwanted consequences from full openness doesn't imply that hiding information or not sharing it can always be justified. In fact, the research enterprise can benefit from higher levels of openness in the sharing of research results and in how research is conducted and accessed, provided that the conditions and processes that promote healthy access to information and that discourage any unwanted or unintended negatives are nurtured and improved. This is the spirit underlying many of the recent policy changes at the Nature research journals.

All Nature-branded journals are now publicly thanking the reviewers who have helped vet and improve the papers that are published by naming them if they have agreed to be identified. And since Autumn 2020, a number of Nature journals have been publishing all reviewer reports and author rebuttal letters, and, in some journals, including Nature Biomedical Engineering, the editorial decision letters, if the authors agreed when their paper was accepted (the reviewers and editors cannot revoke the authors' decision). These peer-review files provide useful context about the improvements to the work during peer review and about the evaluation of the work by the reviewers and journal editors (as exemplified by these three files). However, the files do not include all of the information that was available to (or acquired by) the reviewers and the editors. For some papers, it would be difficult, problematic or unproductive to publish internal written discussions, and any e-mail and voice conversations, for

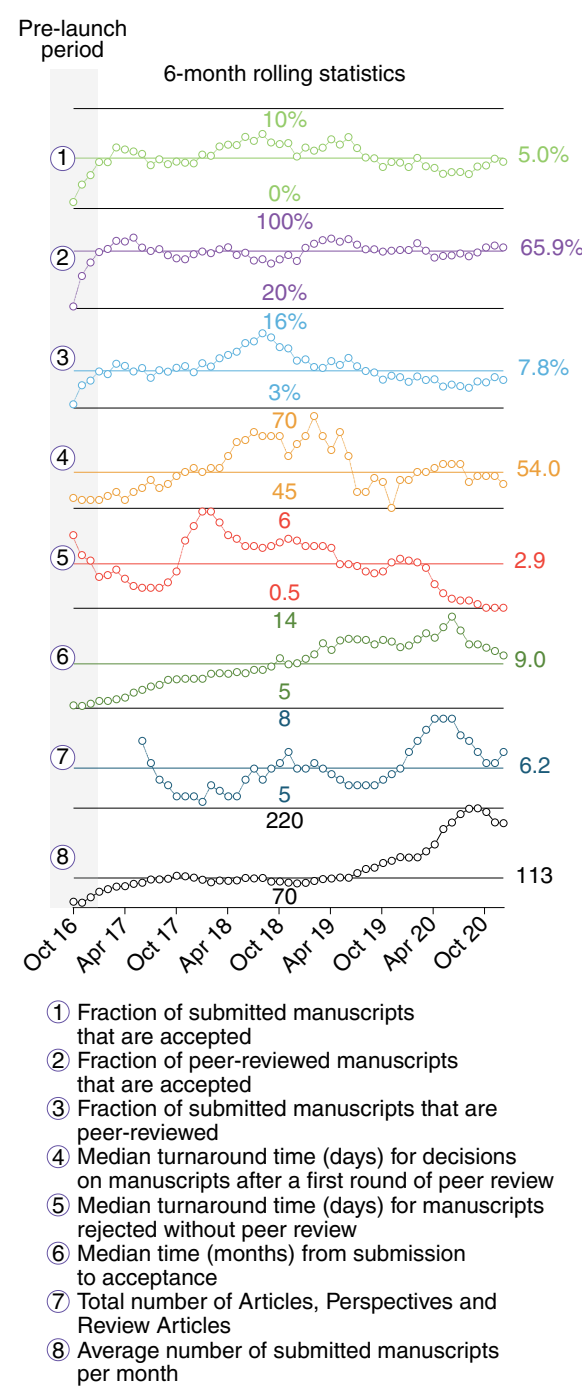

Fig. 1 | Key statistics of Nature Biomedical Engineering since its launch in January 2017. Each dot corresponds to a 6-month window ending at the indicated month. The numbers on the right represent all-time medians. The numbers at the bottom and top of each graph indicate the graphed minimum and maximum values. Fluctuations and trends in the data are a result of a myriad factors, including workload fluctuations owing to editorial projects, travelling commitments and changes in staffing levels. The graph is regularly updated.

the sake of maximum transparency. And authors can have valid reasons to redact some information in the peer-review file and to opt out from having it published. 
For example, discussions during peer review may constrain a patent application, or harm ongoing or future collaborations. Moreover, the Nature journals have long allowed for preprint deposition, and now encourage data and code deposition as well (Nat. Biomed. Eng. 4, 845-846; 2020). And at Nature Biomedical Engineering, we report key rolling metrics (selectivity, turnaround times and publication volumes; Fig. 1) of the service that we provide to the biomedical community. We expect that all of these transparency-increasing initiatives promote trust in peer review (Nat. Biomed. Eng. 4, 663-664; 2020).

Since January 2021, all Nature-branded journals publishing original research (except Nature Communications, which only publishes papers open-access) offer gold open-access publication: authors of primary research can opt to pay an article processing fee (currently $€ 9,500$ ) and have their paper published open-access with a CC-BY license. A few of the journals have started a pilot guided open access - offering publication at one of three journals (for $€ 4,790$ or less), provided that the authors relinquish some control to the editors over which journal their paper will be published in (authors can always walk away from this process after receiving an editorial assessment report, which will include the reviewer assessments, for $€ 2,190$ ). All journals continue to offer closed-access publication based on the traditional subscription model, and to support green open-access publishing with a 6-month embargo. Importantly, editorial decisions won't be influenced by financial and publishing considerations: authors won't be asked to choose the publication route until the paper has been accepted (or before manuscript submission, in the case of guided open access), and the editors of the journals will be shielded from such decisions.

The hefty article processing charges for open access at the Nature journals won't be affordable for most researchers or all funders. Although many authors at well-funded institutions may eventually have these fees covered by transformative agreements between publishers and funders or research institutions, the fact is that, for highly selective journals, open access forces their publishers to spread the cost base across their limited pool of authors rather than the much bigger pool of readers. This will exacerbate inequalities in science publishing. In a predominantly open-access environment, researchers, institutions and funders with more resources will find it increasingly easier to achieve bigger exposure and rewards. And for those whom journal selectivity and certification, as well as the easier and wider accessibility of open-access articles, provide the most value (in further funding, and in any benefits of accrued standing, which may amount to many multiples of the open-access fees paid), making their best papers open access may turn out to be a no-brainer.

Optional and measured (or delayed) openness and transparency may often be wise. From the public eye, there is indeed, sometimes, something to hide.

Published online: 22 January 2021

https://doi.org/10.1038/s41551-021-00686-0 